\title{
Gap waveguides for millimeter wave slow wave structures
}

\author{
Amira Zied Abozied and Rosa Letizia \\ Engineering Department \\ Lancaster University \\ Lancaster, UK
}

\begin{abstract}
The use of groove gap waveguide technology is proposed to aid the design of wide band slow wave structures for millimeter wave travelling wave tubes. The possibility to control the electromagnetic wave without the need for metal contact at the walls can facilitate the realization at high frequencies while increasing design flexibility.
\end{abstract}

Keywords - SWS, millimeter wave TWT, gap waveguide

\section{INTRODUCTION}

The possibility to generate high power in a wide band at millimeter waves $(80-300 \mathrm{GHz})$, has dramatically increased the development of travelling wave tubes (TWT) for this portion of the spectrum. Many are the applications currently driving this effort that will benefit from high power, compact sources at these frequencies, such as satellite and wireless communications, imaging, and healthcare.

Several waveguide configurations have been so far proposed as slow wave structures (SWSs) for the millimeter wave range [1]. At these frequencies, dimensions become very small and waveguide configurations that can alleviate the difficulties of fabrication and assembly while still offering a relatively good interaction with the beam continue to be sought. This becomes especially important to lower the cost of TWTs for applications such as high-capacity wireless communications where the demand for high power at millimeter waves is very high. Among the possible SWSs, the suitability of rectangular corrugated waveguides has been demonstrated via CNC micromachining, LIGA, and DRIE for the millimeter wave and $\mathrm{THz}$ range.

It has been shown that the use of periodic structures exhibiting an electromagnetic bandgap to replace the waveguide walls can aid the design of SWSs and couplers for sheet beam [2]. In particular, their main advantage derives from an increased control over waveguide propagation which can result in easy damping of unwanted modes, integrated filtering capabilities and novel coupler topologies. In this context, gap waveguide technology in the form of ridge gap waveguides and groove gap waveguides has recently emerged as a novel solution to the design of low loss millimeter wave waveguiding. As one type of periodic structure, the gap waveguide retains all the advantages cited above, common to every electromagnetic bandgap structure. In addition, as compared to conventional metal waveguides, gap waveguides control wave propagation through the use of a contactless parallel high impedance surface (an artificial magnetic conductor) and conducting plate. Under the condition that the gap is less than a quarter of the wavelength, no electromagnetic propagation is allowed through this structure unless some guiding element is included in the form of a ridge or a groove waveguide, for example [3-4]. The increased flexibility of this technology with respect to what presented in
[2] can be exploited to facilitate the design and realization of slow wave structures in the millimeter wave range. This work presents initial studies of a gap waveguide SWS for sheet beam TWT at W-band.

\section{GAP WAVEGUIDES SWS}

\section{A. Pin-based Gap waveguide SWS}

The gap waveguide configuration is based on two parallel surfaces, one perfect electric conductor (PEC) and the other an artificial magnetic conductor (AMC), which realize a stop band in frequency. Propagation is permitted within a central groove or ridge realised within the periodic structure. Here, a groove gap waveguide is used to replace the conventional

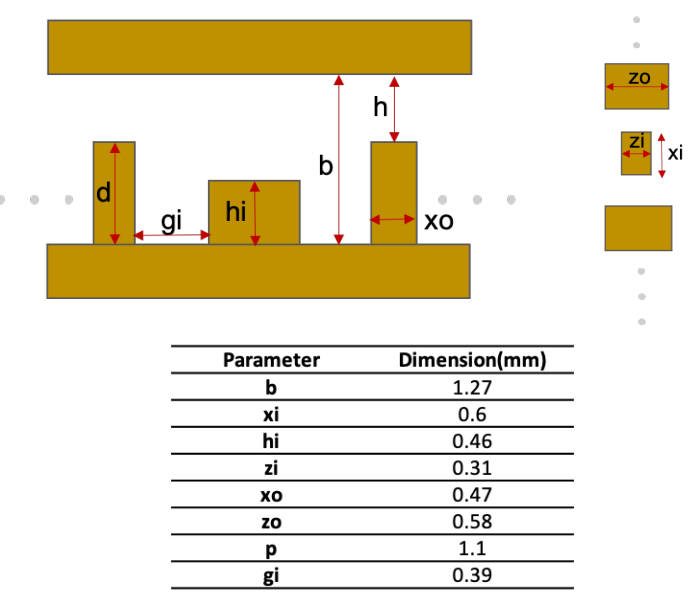

Fig. 1 Schematic of gap waveguide SWS; (a) front view, (b) top view.

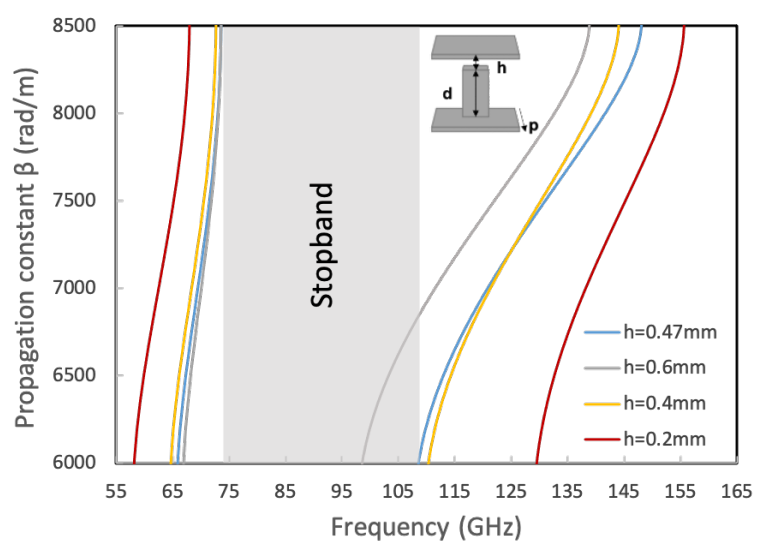

Fig. 2. Band diagram for the AMC-PEC unit cell shown in inset at different gap sizes. The stopband is shown for $h=0.47 \mathrm{~mm}$.

rectangular waveguide enclosure. The groove is loaded longitudinally to realize the hybrid mode required for 
synchronism with the beam. The use of a pin-based surface where the pin length is about a quarter of the wavelength as artificial magnetic conductor has shown to provide wide bandwidth with the advantage to be realised all in metal.

Fig. 1 shows the schematic diagram of the proposed structure. The central groove is filled with a longitudinal corrugation. To realise effective waveguiding, typically 2-3 rows of metal pins at each side of the groove are sufficient. In this context, due to the presence of a metallic enclosure for vacuum realization, even just one pin at each side of the central corrugation can generate the required stopband. Eigenmode simulations are run to optimize the AMC/ PEC unit cell dimensions using CST Microwave Studio [5]. Fig. 2 shows the effect of changing gap size on the width of the stopband. It can be seen that a higher gap size of the pin structure mostly affects the low band edge frequency leading to narrower stopbands. This dimension can be varied depending on the specific realization process for the SWS and required bandwidth. Here, $h=0.47 \mathrm{~mm}$ is chosen to obtain TWT operation in the range $87-100 \mathrm{GHz}$. The complete gap waveguide based SWS (GW-SWS) as in Fig. 1 is then simulated to design the waveguide mode for synchronism. A parametric study of how the beam voltage required for synchronism changes by varying the pin transverse size, xo, is shown in Fig. 3. At $x o=0.47 \mathrm{~mm}$, good overlap of the beam line with the linear region of the mode dispersion is achieved for beam voltage around $18 \mathrm{kV}$. The GW-SWS can only support the operation mode.

\section{B. Half-height pin Gap waveguide SWS}

It has been recently demonstrated that the stopband properties of gap waveguides based on pin structure can be maintained if two roughly half-height pin structures are considered as top and bottom plate. The gap is taken as the distance between these half-height pin surfaces [6]. This topology makes the realization of the pin structure significantly easier and more suitable to high frequency. The structure is also robust to marginal misalignment between top and bottom structure, which is important to maintain an easy assembly of the SWS. A half-height gap waveguide based SWS (HGW-SWS) has been designed for comparison. To match dispersion characteristic of the waveguide mode, the total length of the pin, $d$, is changed to $1.1 \mathrm{~mm}$ with a gap size $h=0.17 \mathrm{~mm}$. Assuming a sheet beam with size $0.8 \times 0.2 \mathrm{~mm}$ is used at a distance of $60 \mu \mathrm{m}$ from the top of the central corrugation, the average coupling impedance for both the GW-SWS and HGW-SWS has been calculated and compared in Fig. 4. It can be noted that the values closely overlap in the bandwidth of interest and are comparable to other similar corrugated waveguides in the literature.

\section{CONCLUSIONS}

Two configurations of gap waveguide-based SWS have been proposed for the design of W-band TWTs. Depending on the required frequency of operation and fabrication technology, gap waveguides can facilitate the design and realization of suitable SWSs.

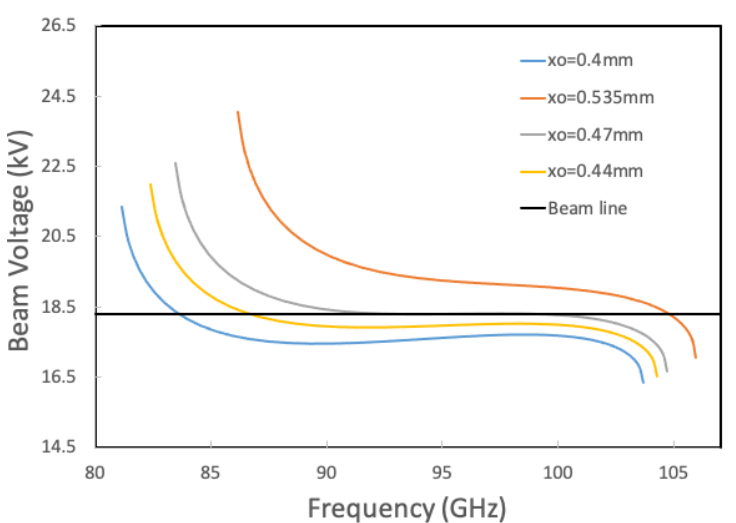

Fig. 3. Beam voltage for varying pins transverse size, $x o$. The black line shows synchronism for beam voltage $\sim 18 \mathrm{kV}$ for $x o=0.47 \mathrm{~mm}$.

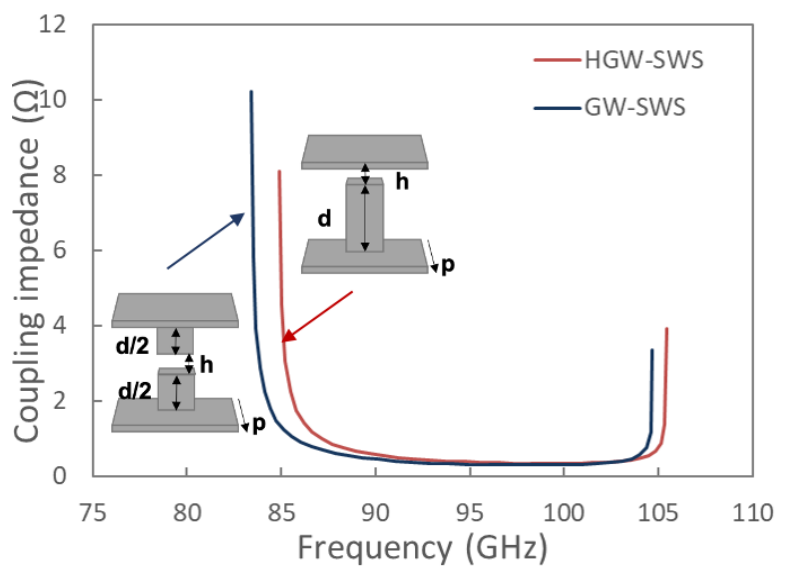

Fig. 4. Coupling impedance comparison for the HGW and the GWSWS. A schematic of the pin configuration for both structures is shown in inset.

\section{ACKNOWLEDGMENT}

Rosa Letizia's Fellowship was supported by the Royal Academy of Engineering under the Leverhulme Trust Senior Fellowship scheme.

\section{REFERENCES}

[1] C. Paoloni, D. Gamzina, R. Letizia, Y. Zheng and N. C. Luhmann Jr., "Millimeter Wave Traveling Wave Tubes for the 21st Century", J. Electromagn. Wav. Appl., Dec. 2020. https://doi.org/10.1080/09205071.2020.1848643

[2] R. Letizia, M. Mineo and C. Paoloni, "Photonic Crystal-Structures for $\mathrm{THz}$ Vacuum Electron Devices," in IEEE Transactions on Electron Devices, vol. 62, no. 1, pp. 178-183, Jan. 2015, doi: 10.1109/TED.2014.2366639.

[3] P. Kildal, E. Alfonso, A. Valero-Nogueira and E. Rajo-Iglesias, "Local Metamaterial-Based Waveguides in Gaps Between Parallel Metal Plates," in IEEE Antennas and Wireless Propagation Letters, vol. 8, pp. 84-87, 2009, doi: 10.1109/LAWP.2008.2011147.

[4] E. Tahanian and G. Dadashzadeh, "A Novel Gap-Groove FoldedWaveguide Slow-Wave Structure for G-Band Traveling-Wave Tube," in IEEE Transactions on Electron Devices, vol. 63, no. 7, pp. 29122918, July 2016, doi: 10.1109/TED.2016.2564740.

[5] CST Microwave Studio www.cst.com

[6] F. Fan, J. Yang, V. Vassilev and A. U. Zaman, "Bandwidth Investigation on Half-Height Pin in Ridge Gap Waveguide," in IEEE Transactions on Microwave Theory and Techniques, vol. 66, no. 1, pp. 100-108, Jan. 2018, doi: 10.1109/TMTT.2017.2732983. 